\title{
Autophagy as a Physiological Response of the Body to Starvation
}

\author{
Secil Erbil-Bilir, Devrim Gozuacik, and Ozlem Kutlu
}

\begin{abstract}
Macroautophagy is a cellular degradation pathway that deliver cytoplasmic components such as damaged organelles, misfolded proteins, and pathogens to the lysosomes for degradation. Autophagy is associated with the survival of the cell under stress conditions and infections. Nutrient deprivation is one of the main inducers of autophagy, which recycles cytoplasmic components to provide building blocks required for cell survival and maintains cellular homeostasis. Due to its cytoprotective effects, autophagic responses are necessary in resisting diseases and ensuring health. Understanding the regulation of autophagic responses in mammalian cells is required to improve human health through innovations in treatment strategies. This chapter focuses on recent findings about autophagy mechanisms and their role in the body's response to starvation as well as the current knowledge of autophagy-related malnutrition disorders.
\end{abstract}

\section{Keywords}

Autophagy • Starvation • Stress • mTOR • AMPK • LC3 • ULK1 • ATG •

Metabolic disease $\cdot$ Lysosome

S. Erbil-Bilir

Molecular Biology, Genetics and Bioengineering Program, Sabanci University, Istanbul, Turkey

e-mail: secilerbil@sabanciuniv.edu

D. Gozuacik

Molecular Biology, Genetics and Bioengineering Program, EFSUN-Center of Excellence for Functional Surfaces and Interfaces for Nano Diagnostics, Sabanci University, Istanbul, Turkey e-mail: dgozuacik@sabanciuniv.edu

O. Kutlu $(\bowtie)$

Nanotechnology Research and Application Center, EFSUN-Center of Excellence for Functional Surfaces and Interfaces for Nano Diagnostics, Sabanci University, Istanbul, Turkey

e-mail: ozlemkutlu@sabanciuniv.edu 


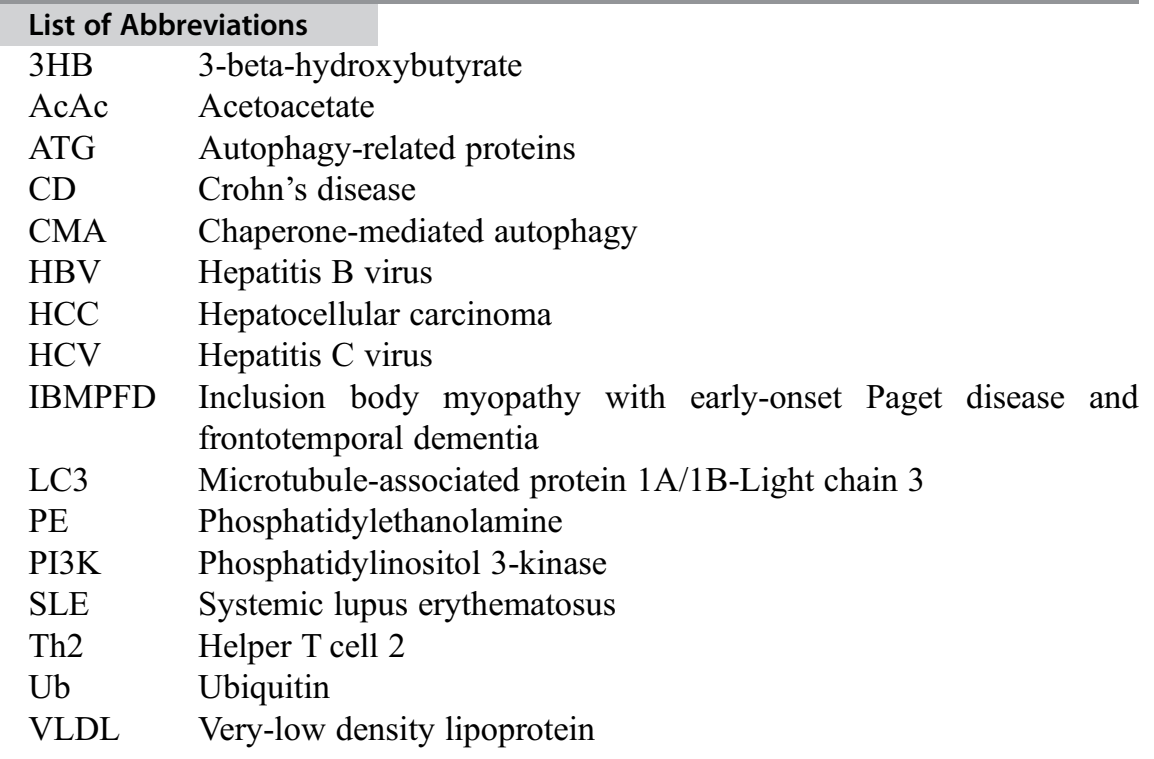

\section{Contents}

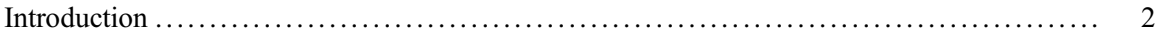

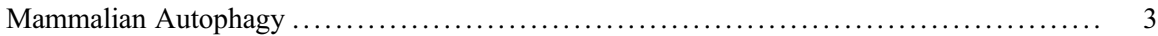

Molecular Signaling Pathways in Starvation-Induced Autophagy $\ldots \ldots \ldots \ldots \ldots \ldots \ldots \ldots \ldots, \quad 3$

Autophagy at Organ and Tissue Level and Affected Human Diseases from Their Impairment 6

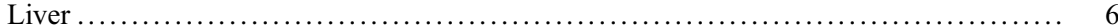

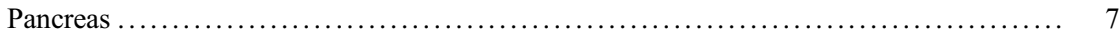

Muscular Tissue ......................................................... 8

Other Human Diseases Affected by Mutations in Starvation-Induced Autophagy Genes ..... 8

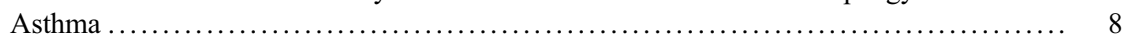

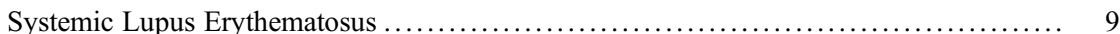

Crohn's Disease . . . . . . .

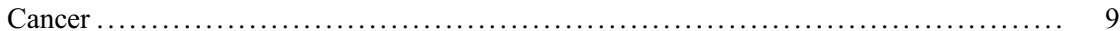

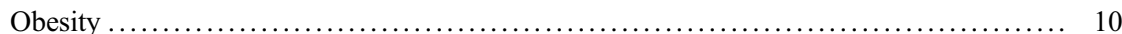

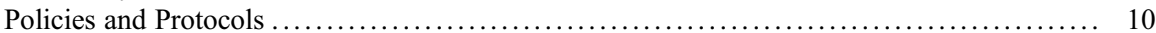

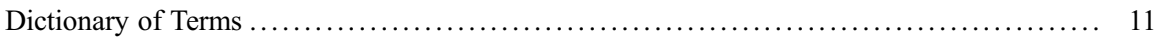

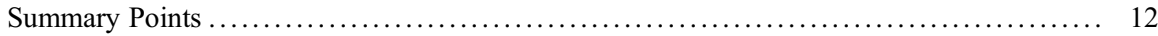

References ............................................................. 13

\section{Introduction}

Autophagy is the primary cellular response of cells to starvation. In mammalian cells, survival responses are mainly dependent on hormonal and neuronal deregulations from stress signals. Mammalian cells respond to starvation by activating lipolysis and proteolysis, which are regulated by autophagic activation pathways upon nutrient deprivation (Hosokawa et al. 2009; Petibone et al. 2017). Activation of lipolysis is required to provide fatty acids through the breakdown of triacylglycerols 
as an energy source for different tissues and organs during nutrient deprivation (Cockburn and Coore 1995; Angelini et al. 2016). Moreover, glycerol, which is a side product of lipolysis, can be utilized in the liver to be used as a glucose source under starvation conditions. When cells are exposed to long-term nutrient deprivation and energy loss, an excess amount of lipolysis results in the production of ketone bodies, which are the end product of free fatty acid oxidation reaction and can be used as an energy source in muscle and neurons. Since not all cells have the ability to use ketone bodies as an energy source, the cells that cannot utilize these small molecules activate proteolysis as a response to starvation (Luo et al. 2017; Lee et al. 2017). Proteolysis can be activated in two different ways, depending on the tissue type and stress duration. The first is the ubiquitin-mediated proteolysis system, which selectively degrades the nonfunctional or misfolded proteins which are linked to ubiquitin markers. Ubiquitin-mediated proteolysis is mostly active in skeletal muscle cells. The second pathway is the lysosomal pathway, which is activated upon long-term starvation and is responsible for the nonselective degradation of proteins through enzymatic activity in lysosomes.

\section{Mammalian Autophagy}

Autophagy is a well conserved lysosomal catabolic pathway that is required for the recycling of cellular compartments and proteins. However, this chapter is focused on macroautophagy, which is the predominant form in mammals. There are two additional types of autophagy: microautophagy and chaperone-mediated autophagy (CMA). Microautophagy occurs via the direct engulfment of cytoplasmic cargo by lysosomes by which it is degraded by enzymatic activity. CMA is a selective type of autophagy which occurs by chaperone-mediated selection and direct translocation of proteins into lysosomes without any need for the formation of double membrane structures. Macroautophagy (hereafter referred to as autophagy) is mostly activated upon stress signals in mammals; however, it also occurs under normal conditions in order to maintain cellular homeostasis by providing the turnover of long-lived, damaged, nonfunctional proteins in the cell (Erbil et al. 2016; Mathiassen et al. 2017). Upon stress, autophagy starts with the initiation and elongation of the little membrane structures in the cytosol. These double membrane structures engulf the cargo and form the autophagosomes. Lysosomes are then fused with the autophagosomes in order to form autolysosomes, by which the cargo is degraded by lysosomal enzymes and byproducts are released back to the cytosol to be utilized as an energy supply in the cell.

\section{Molecular Signaling Pathways in Starvation-Induced Autophagy}

Identification of the autophagy-related (ATG) proteins leads to an improvement of the clarification of the molecular signaling pathways of autophagy. Recently, 36 ATG proteins have been discovered in the core machinery of mammalian autophagy. These proteins are involved in different functional complexes in autophagycascade. 


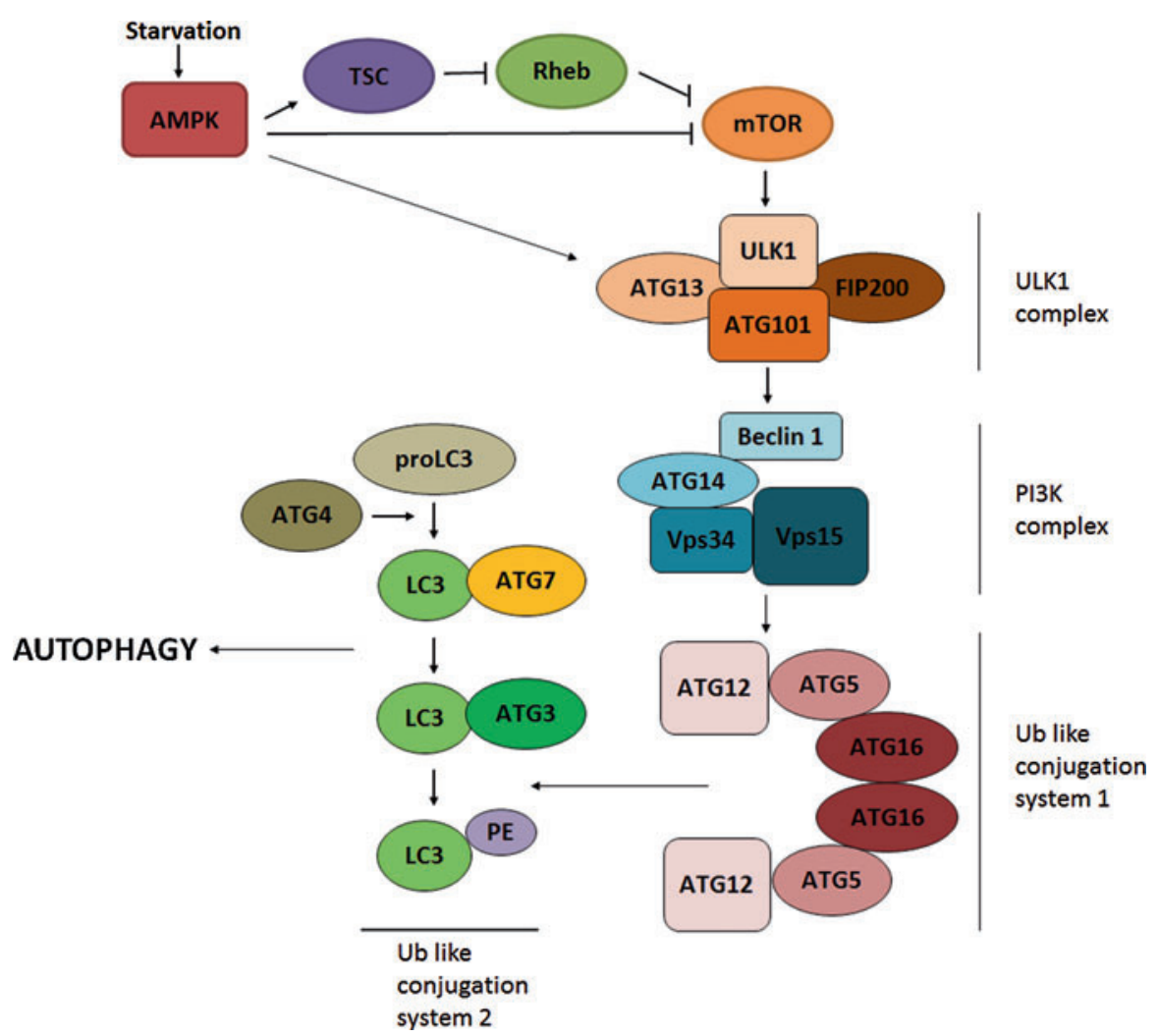

Fig. 1 Signaling regulation of starvation-induced autophagy. In mammals, starvation-induced autophagy starts with the activation of AMPK and TSC followed by the inhibition of Rheb and mTOR through phosphorylation events. When nutrients are limited, the phosphorylation of ULK1 by mTOR is inhibited due to mTOR inactivation. Upon dephosphorylation, ULK1 can interact with the other complex elements which mediate the formation of PAS leading to the initiation of autophagosome structures. Further nucleation steps require a PI3K complex which is formed by VPS34 and VPS15 and their regulatory subunits Beclin 1 and ATG14. Membrane elongation and closure requires two different Ub-like conjugation systems. Ub-like conjugation systems 1 and 2 together lead to PE conjugation of LC3 that is essential for the elongation of the membrane structures. Moreover, membrane-bound LC3 is required for the recognition of the cargo through adaptor proteins as well as the lysosome fusion to the autophagosomes. The resulting structure is named as autolysosomes and the cargo is degraded by enzymatic activity inside these structures. Key: PAS, phagophore assembly site; TSC, tuberous sclerosis complex; VPS34 and 15, vacuolar protein sorting 34 and 15; Ub, ubiquitin; PE, phophatidylethanolamine; LC3, microtubule-associated protein 1 light chain 3

The main four complexes are demonstrated in Fig. 1: ULK1 and phosphatidylinositol 3 kinase (PI3K) complexes, Ubiquitin (Ub)-like conjugation systems 1 and 2. Table 1 summarizes their key roles in the activation of autophagy as the cellular response to starvation in mammals. 
Table 1 Key protein complexes acting in starvation-induced autophagy in mammalian cells. ULK1 complex, PI3K complex, Ub-like conjugation system 1, and Ub-like conjugation system 2 are the key protein complexes that act in starvation-induced autophagy. ULK1 complex consists of ULK1, ATG13, FIP200, and ATG101; PI3K complex consists of Beclin1, ATG14, Vps34, and Vps15; Ub-like conjugation system 1 consists of ATG5, ATG12, and ATG16; Ub-like conjugation system 2 consists of ATG3, ATG4, ATG7, and LC3 proteins. Key: PI3K, phosphatidylinositol 3 kinase; ATG3, 4, 5, 7, 12, 13, 14, 16, and 101, autophagy-related proteins 3, 4, 5, 7, 12, 13, 14, 16, and 101; VPS34 and 15, vacuolar protein sorting 34 and 15; Ub, ubiquitin; LC3, microtubuleassociated protein 1 light chain 3

\begin{tabular}{l|l|l}
\hline Complex & $\begin{array}{l}\text { Complex } \\
\text { elements }\end{array}$ & Function \\
\hline ULK1 complex & $\begin{array}{l}\text { ULK1 } \\
\text { ATG13 } \\
\text { FIP200 } \\
\text { ATG101 }\end{array}$ & $\begin{array}{l}\text { This complex is negatively regulated by mTOR. Upon } \\
\text { autophagy induction by starvation signals received by } \\
\text { mTOR, ULK1 is activated by self-phosphorylation and } \\
\text { translocates into the early autophagic structures. In addition } \\
\text { to its self-phosphorylation, ULK1 phosphorylates ATG13 } \\
\text { and FIP200 due to complex activation }\end{array}$ \\
\hline PI3K complex & $\begin{array}{l}\text { Beclin 1 } \\
\text { ATG14 } \\
\text { Vps34 } \\
\text { Vps15 }\end{array}$ & $\begin{array}{l}\text { This complex is negatively regulated by Bcl-2 Beclin 1 } \\
\text { interaction. Upon autophagy induction by starvation signals, } \\
\text { Bcl-2 disassociates from Beclin 1 and forms the complex } \\
\text { with other elements on the ER membrane. This complex is } \\
\text { effective in the later autophagic events, as the inhibition of } \\
\text { this complex result in the impairment of autophagosome and } \\
\text { lysosome fusion }\end{array}$ \\
\hline $\begin{array}{l}\text { Ub-like } \\
\text { conjugation } \\
\text { system 1 }\end{array}$ & $\begin{array}{l}\text { ATG5 } \\
\text { ATG12 } \\
\text { ATG16 }\end{array}$ & $\begin{array}{l}\text { This complex is necessary for LC3 and } \\
\text { phophatidylethanolamine (PE) conjugation which is } \\
\text { essential for the elongation of the membrane structures }\end{array}$ \\
\hline $\begin{array}{l}\text { Ub-like } \\
\text { conjugation } \\
\text { system 2 }\end{array}$ & $\begin{array}{l}\text { ATG3 } \\
\text { ATG4 } \\
\text { ATG7 } \\
\text { LC3 }\end{array}$ & $\begin{array}{l}\text { Deconjugation of LC3 form ATG4 is important for the LC3- } \\
\text { PE formation and the closure of the autophagosome } \\
\text { membrane. Since LC3-PE is localized both in the inner and } \\
\text { the outer membranes of the autophagosome, its formation by } \\
\text { Ub-like conjugation system 2 is important for the adaptor } \\
\text { protein binding, such as p62, for selective autophagy }\end{array}$ \\
\hline
\end{tabular}

AMPK is the main energy sensor of the cell, and it plays a crucial role in the induction of starvation-induced autophagy in mammalian cells (Garcia and Shaw 2017). When a nutrient level is limited, AMPK activation leads to mTOR inhibition through TSC activation and Rheb inhibition. Therefore, AMPK indirectly activates the ULK1 complex which is in the downstream of mTOR and kept inactivated through phosphorylation events. Recent studies have showed that in addition to mTOR, AMPK can also phosphorylate ULK1 directly to lead to its activation upon starvation signals. Following phosphorylation, ULK1 interacts with the other complex elements, FIP200, ATG101, and ATG13, to form the ULK1 complex and phosphorylates them to activate the complex (Petherick 2015). The active ULK1 complex translocates to the ER membrane and activates the PI3K complex which consists of Beclin 1, ATG14, and vacuolar protein sorting 34 and 15 (VPS34 and 15). The PI3K complex recruits Ub-like conjugate system 1, formed by ATG12-5-16 proteins, which plays a role in the elongation and closure of the isolation membrane (Zhong et al. 2017). Moreover, Ub-like conjugate system 1 is also required for the 
activation of $\mathrm{Ub}$ like conjugate system 2, which is necessary for phosphatidylethanolamine (PE) conjugation to LC3 (microtubule-associated protein 1A/1B-light chain 3) protein. The cytosolic form of LC3 is called pro-LC3. Due to the induction of autophagy, pro-LC3 is cleaved by ATG4, and then the cleaved form of LC3 is conjugated with PE by the activities of E1-like enzyme ATG7 and E2-like enzyme ATG3. PE-conjugated LC3 is membrane-bound, and it is present both in the inner and outer sides of the autophagosome (Nath et al. 2014). Therefore, it not only plays a role in membrane elongation and closure but also serves as the recognition site for adaptor proteins such as p62 (Klionsky et al. 2010).

\section{Autophagy at Organ and Tissue Level and Affected Human Diseases from Their Impairment}

Dysregulation of autophagy has been implicated in a range of diseases. Starvationinduced autophagy impairments mostly cause liver, pancreas, and muscular tissue disorders (summarized in Fig. 2).

\section{Liver}

Basal autophagy is required to maintain liver homeostasis through the clearance of protein aggregates and damaged mitochondria in order to prevent hepatocyte swelling (Wang et al. 2015). Liver autophagy is mainly controlled by hormones. While insulin, aminoacids, and glucose inhibit liver autophagy, glucagon activates the process. Activation of autophagy contributes to lipophagy through fatty acid $\beta$ oxidation, ketone body production, and glucogenesis in the liver. Moreover, liver autophagy is involved in the control of very-low-density lipoprotein (VLDL) and plasma glucose concentrations upon nutrient deprivation (Sparks et al. 2013). Since liver and liver autophagy has an extremely important role in homeostasis, the impairment of autophagy in this organ causes vital disorders such as alcoholic liver disease, fatty liver disease, hepatocellular carcinoma (HCC), liver fibrosis, and viral infection in the liver (Niu et al. 2016).

Alcohol consumption activates autophagy in the liver through the AMPK pathway in hepatocytes (Wang et al. 2016). Autophagy activation leads to the removal of lipid droplets and damaged mitochondria, thus preventing cell death. However, if alcohol consumption becomes chronic, excess amounts of ethanol inhibit hepatocellular autophagy by blocking both AMPK activation and vesicular transport that is required for autophagosome formation (Cho et al. 2017). As a result, the accumulation of aggregated proteins and damaged mitochondria leads to cell death. Additionally, lipid droplets that cannot be removed through lipophagy causes steatosis and fatty liver disease.

Hepatitis $\mathrm{B}$ and $\mathrm{C}$ viruses (HBV and $\mathrm{HCV}$, respectively) require autophagy for their replication in hepatocytes. The viral proteins activate the PI3K complex and 


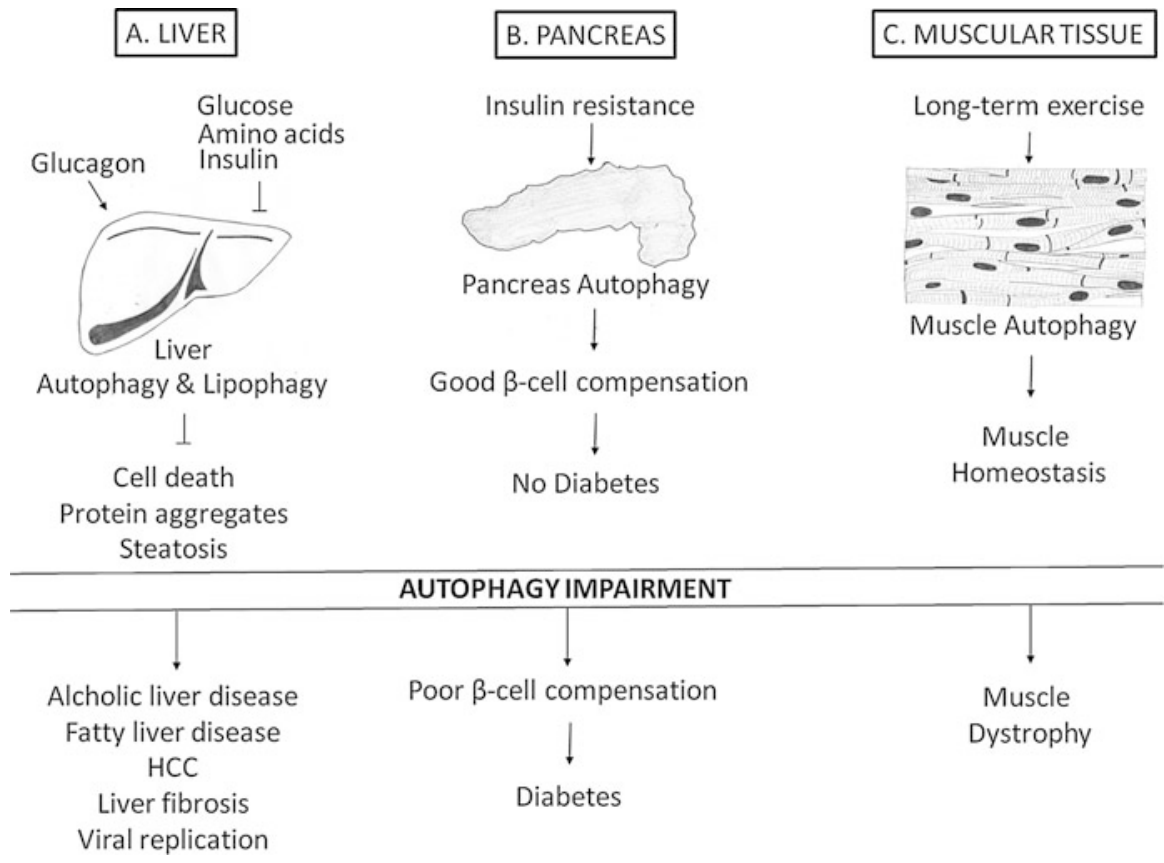

Fig. 2 The role of autophagy in liver, pancreas, and muscular tissue disorders. (a) Liver autophagy is inhibited by glucose, insulin, and amino acids under normal conditions. It is induced as the cytoprotective mechanism and increased glucagon level which lead to the inhibition of cell death, steatosis, and the accumulation of protein aggregates. Impaired liver autophagy may result in alcoholic and fatty liver disease, HCC, liver fibrosis, or viral replication in the liver. (b) Pancreatic autophagy is activated against insulin resistance to prevent poor $\beta$-cell compensation and diabetes. Since autophagy-defective cells cannot compensate the $\beta$-cells, diabetes occurs as a result of insulin resistance. (c) Muscle autophagy is activated after long-term exercise and maintain muscle cell homeostasis. Autophagy impairment in the muscular tissue result in macular dystrophy. Key: HCC, hepatocellular carcinoma

trigger autophagosome formation for their benefits (Vescovo et al. 2016). Therefore, regulation of autophagy in the liver is vital for viral infections that may end up with HCC.

\section{Pancreas}

Pancreatic $\beta$-cells are responsible for the storage and release of insulin (Morita et al. 2017). Pancreas autophagy has a significant role in maintaining $\beta$-cell homeostasis by keeping their mass and function properly. Impairment of autophagy results in dysfunctional $\beta$-cell and type- 2 diabetes which is characterized by insulin resistance. In vivo studies in ATG7 -/- mice have revealed that autophagy-defective $\beta$-cells lose their mass and insulin content, which causes suppressive insulin release (Rivera et al. 2014). Moreover, since autophagy is necessary for the clearance of damaged 
mitochondria, impaired autophagy in pancreatic $\beta$-cells leads to the rise of insulin resistance and impaired glucose tolerance (Xu et al. 2016).

\section{Muscular Tissue}

Similar to other organs, in muscle cells, autophagy is vital for organelle and protein aggregate clearance. However, excess amount of autophagy activation cause muscle weight loss. Impaired autophagy was also found as a main reason of malfunctional and dystrophic muscles after long-term exercise or starvation (Fiacco et al. 2016).

Autophagy is one of the major pathways of muscle homeostasis. It preserve myofiber integrity and prevents muscle damage. Autophagy impairment has been reported in several muscle diseases such as inclusion body myopathy with earlyonset Paget disease and frontotemporal dementia (IBMPFD) (Bayraktar et al. 2016), Pompe Disease (Lim et al. 2017), and Danon Disease (Nascimbeni et al. 2017) (please see "Dictionary of Terms" section). In ATG7 null muscle cells obtained from ATG7-/- mice, distension of sarcoplasmic reticulum and random nonfunctional membrane structure accumulations were demonstrated (Mizushima and Komatsu 2011). All these effects may be the reason for muscular dystrophies which directly result in patient death due to myofibre necrosis in respiratory and cardiac systems.

\section{Other Human Diseases Affected by Mutations in Starvation- Induced Autophagy Genes}

Emerging molecular techniques and accumulated data about autophagy pathways allow researchers to carry out specific analyzes. Therefore, the involvement of autophagy, particularly on the molecular level, in human diseases is an attractive topic for clinical research. These diseases include asthma, systemic lupus erythematosus (SLE), Crohn's disease (CD), several types of cancers, and obesity (The diseases found to be directly related to the starvation-induced autophagy gene mutations are listed in Table 2.).

\section{Asthma}

Asthma is a common respiratory tract inflammation disease that causes high rates of death all over the world. Using a mice model, in vivo studies have showed that autophagy has a role in the clearance of pathogens in canonical ways, as well as its effect on helper T cell 2 (Th2) activation due to infection. Moreover, polymorphisms in ATG5 gene result in asthma symptoms in mice models (Martin et al. 2012; Pham et al. 2016). 
Table 2 Known human diseases affected by mutations in starvation-induced autophagy genes. Impairments of starvation-induced autophagy resulted from ATG5 polymorphism, ATG16L T300A mutation, Beclin1 monoallelic deletion, mutations in PI3K and TSC1, or loss of ATG7 are the known causes of human diseases such as asthma, systemic lupus erythematosus, several types of cancer, and obesity. Key: ATG5, 7, and 16L, autophagy-related proteins 5, 7 and 16 like 1; T300A, threonine300 mutation to alanine; PI3K, phosphatidylinositol 3 kinase; TSC1, tuberous sclerosis complex 1

\begin{tabular}{l|l}
\hline Mutation & Associated human disease \\
\hline ATG5 polymorphism & $\begin{array}{l}\text { Asthma (Martin et al. 2012), systemic lupus erythematosus } \\
\text { (Zhou et al. 2011) }\end{array}$ \\
\hline ATG16L T300A mutation & Crohn's disease (Massey and Parkes 2007) \\
\hline Beclin 1 monoallelic deletion & $\begin{array}{l}\text { Breast, ovarian, colorectal, and prostate cancers (Liang et al. } \\
\text { 1999) }\end{array}$ \\
\hline $\begin{array}{l}\text { Gain of function mutations in } \\
\text { PI3K }\end{array}$ & Cancer (Cully et al. 2006) \\
\hline TSC1 mutation & $\begin{array}{l}\text { Benign hamartomatous growths in multiple organs (Schwartz et } \\
\text { al. 2007) }\end{array}$ \\
\hline Loss of ATG7 & Obesity (Yang et al. 2010) \\
\hline
\end{tabular}

\section{Systemic Lupus Erythematosus}

Systemic lupus erythematosus (SLE) is an autoimmune disease that is described by the self-reaction of the immune system to healthy tissues and organs (Gavand et al. 2017). The pathogenesis of the disease is not clear yet; however, $50 \%$ of patients are affected genetically. In the Chinese population, rare variants ATG5 were shown to be linked to SLE. In other words, one of the reasons for SLE disease is ATG5 polymorphism (Zhou et al. 2011). Therefore, autophagy proteins could be a potential therapeutic target for SLE treatment.

\section{Crohn's Disease}

Crohn's disease (CD) is an inflammatory bowel disease characterized by multiple gastrointestinal pathology from mouth to anus (Stappenbeck et al. 2011). However, it is not classified as an autoimmune disease; it is an immune system disorder. Although genetic and environmental factors, infections, and defective autophagy are reported in CD, the causal etiologic factors are still unknown. Analyzing $735 \mathrm{CD}$ patients' genotypes in comparison to healthy controls showed that the T300A mutation of ATG16 protein is responsible for Crohn's disease (Massey and Parkes 2007). However, it is not the only reason for CD.

\section{Cancer}

Genetic link between cancer and autophagy has been shown in various cancer types, including human breast, prostate, colorectal, and ovarian cancer (Sumis et al. 2016). 
Most of the studies have showed that Beclin 1 gene is monoallelically deleted in most of the cancer types (Liang et al. 1999). Also, PI3K mutations cause tumor formation and TSC1 mutation leads to benign hamartomatous growths in multiple organs (Cully et al. 2006; Schwartz et al. 2007). As an evident to anticancer effect of autophagy, several studies have reported that the overexpression of ATG genes in cancer cells lead to tumor suppression (Wesselborg and Stork 2015). Although, autophagy is indeed a tumor suppressor pathway, the mechanisms and molecules playing part behind it remain largely unclear.

\section{Obesity}

Obesity is the medical condition, in which the body's fat rate is too much that negatively affects the body health (Yilmaz 2017). It is an excess amount of energy flow which disrupts the energy sensing and the utilizing mechanisms of the body. The role of autophagy in obesity was first studied in mice hepatocytes (Yang et al. 2010). In the study, obese mice hepatocytes represent low levels of ATG7 expression, while the restoration of ATG7 resulted in elevated insulin levels (Zhang et al. 2009). However, it is known that environmental conditions trigger tendency to obesity, which seems to lead autophagy impairments.

\section{Policies and Protocols}

Measuring autophagic activity at cellular level is crucial to identify therapeutic targets for diseases affected by autophagy impairments. In order to detect autophagy activation or inhibition, several molecular techniques are developed based on immunoblotting, immunofluorescence, or isotope release as described in Table 3.

Detection of autophagosomes and autolysosomes by electron microscopy can be used as a conventional autophagy procedure. By using immunoblotting technique, the conversion of cytosolic nonlipidated LC3-I into membrane-bound lipidated form of LC3-II is commonly used for analyzing autophagy induction. However, LC3 is not the direct target of autophagy, as it is degraded in autolysosomes after long-term autophagy. Therefore, LC3 turnover assay is carried out using autophagy inhibitors in order to estimate the level of LC3 protein degradation. Similarly, the GFP-tagged LC3 protein is overexpressed in mammalian cells and the amount of generated free GFP protein can be measured for autophagic activity estimation.

By using immunofluorescence, counting the average number of GFP-LC3 punctate structures on the autophagosome membranes or endogenous LC3 protein are other well-known autophagy techniques. RFP-GFP LC3 protein construct can be used to distinguish the number of autophagosomes and autolysosomes, since GFP signal is quenched by the acidic environment of autolysosomes.

Lastly, long-lived protein degradation can be measured by tracing the radioactive isotope released from the cells indicating active autophagy state. 
Table 3 Protocols for detecting autophagy in mammalian cells. Immunoblotting, immunofluorescence, and isotope release are the three main protocols for detecting autophagy in mammalian cells. LC3 conversion, LC3 turnover, and GFP-LC3 cleavage tests can be carried out and the amount of p62 in the cell can be detected by using immunoblotting procedures. Number of LC3 puncta/cell, RFP-GFP LC3 color change, and the amount of p62 in the cell can be measured by using immunofluorescence techniques; while lysosome-dependent long-lived protein degradation tests can be carried out by using isotope release protocols. Key: LC3, microtubule-associated protein 1 light chain 3; GFP, green fluorescent protein; RFP, red fluorescent protein

\begin{tabular}{l|l|l}
\hline Immunoblotting & Immunofluorescence & Isotope release \\
\hline $\begin{array}{l}\text { 1. LC3 conversion } \\
\text { 2. LC3 turnover }\end{array}$ & $\begin{array}{l}\text { 1. Number of LC3 } \\
\text { puncta/cell }\end{array}$ & $\begin{array}{l}\text { 1. Lysosome-dependent long-lived protein } \\
\text { degradation }\end{array}$ \\
the cell & $\begin{array}{l}\text { 2. Amount of p62 in the } \\
\text { cell }\end{array}$ & \\
4. GFP-LC3 cleavage & $\begin{array}{l}\text { 3. RFP-GFP LC3 color } \\
\text { change }\end{array}$ & \\
\hline
\end{tabular}

\section{Dictionary of Terms}

- Cellular stress - Anything that creates a challenge or a constraint to cell health. Cellular stress can be intrinsic, such as DNA damage, misfolded protein accumulation, and damaged organelles; or extrinsic, such as starvation, toxins, pathogens, toxins, heat, etc.

- Danon disease - An X-linked glucagon storage disorder that is characterized by weakened skeletal and cardiac muscle combined with mental retardation.

- Glucagon - The hormone that is produced and secreted by pancreatic $\alpha$-cells. Its role is opposite to insulin, as it increases the glucose levels in the blood.

- Glucogenesis - The production of glucose by the breakdown of glycogen that is stored in the liver.

- Homeostasis - Disposition of a physiological system to preserve its internal stability and to keep vital functions active

- IBMPFD - Inclusion body myopathy with early-onset Paget disease and frontotemporal dementia, which is a rare multisystem disease characterized by the weakness of muscles and bones in combination to intellectual disability.

- Insulin - The hormone that is produced in pancreatic $\beta$-cells and secreted into blood stream where it meets with glucose and enables it to enter the cells where needed. If glucose level is higher than needed in the blood, pancreatic $\beta$-cells produce and secrete more insulin in order to store the excess amount of glucose. Therefore, insulin is the key hormone that keeps blood sugar level stable.

- Insulin resistance - The pathological condition in which the cells do not recognize insulin and the blood sugar cannot be utilized by the cells. As a result of insulin resistance, the blood sugar level gets higher than the normal.

- Ketone bodies - The water-soluble molecules that are produced in the liver, as the end product of fatty acid oxidation due to energy limitation in the body. There are two main types of ketone bodies: acetoacetate and 3-beta-hydroxybutyrate 
and the spontaneous breakdown product of them is acetone which can be counted as the third type of ketone bodies that are present in the blood.

- Lipolysis - Breakdown of lipid molecules by the hydrolysis of triglycerides to free fatty acids and glycerol.

- Pompe disease - A genetically inherited glucose storage disorder that result in the accumulation of glycogen in muscle cells disabling them to function normally.

- Proteolysis - Breakdown of proteins into aminoacids or smaller aminoacids by protease enzyme activity.

\section{Summary Points}

- This chapter focuses on the recent findings about autophagy mechanism and its role in the body response to the starvation as well as the current knowledge of autophagy-related malnutrition disorders.

- Autophagy is a cellular degradation pathway that deliver cytoplasmic components such as damaged organelles, misfolded proteins, and pathogens to the lysosomes.

- Autophagy is associated to the survival of the cell under stress conditions and infections. The role of autophagy in cell survival is summarized in Fig. 3.

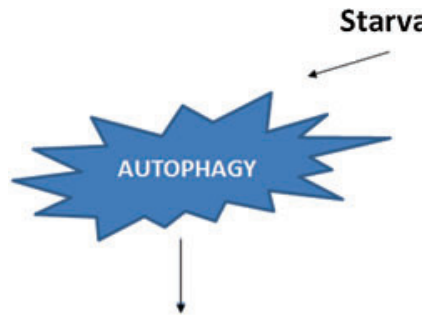

- Stress adaptation by nutrient recycling

- Cellular remodelling

- Supression of apoptosis

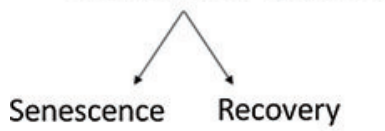

Senescence Recovery
IMPAIRED

AUTOPHAGY

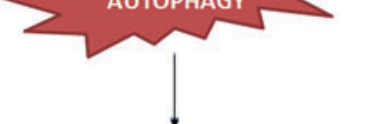

Apoptosis

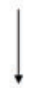

Cell death

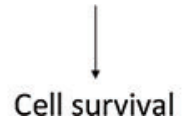

Fig. 3 The role of autophagy in cell survival. (Left) Autophagy acts as a survival mechanism of the cell supporting the cellular adaptation under stress conditions by nutrient recycling and cellular remodeling; (Right) impaired autophagy result in the rapid cell death since apoptotic pathway cannot be inhibited 
- Nutrient deprivation is one of the main inducers of autophagy that recycle cytoplasmic components to provide building blocks required for cell survival and maintain cellular homeostasis

- Autophagic responses are necessary for resisting diseases and maintaining health.

- Understanding the regulation of autophagic responses in mammalian cells are required for improving human health by innovations in treatment strategies.

\section{References}

Angelini C, Nascimbeni AC, Cenacchi G, Tasca E (2016) Lipolysis and lipophagy in lipid storage myopathies. Biochim Biophys Acta 1862(7):1367-1373. doi:10.1016/j.bbadis.2016.04.008

Bayraktar O, Oral O, Kocaturk NM et al (2016) IBMPFD disease-causing mutant VCP/p97 proteins are targets of autophagic-lysosomal degradation. PLoS One 11(10):e0164864. doi:10.1371/ journal.pone. 0164864

Cho HI, Seo MJ, Lee SM (2017) 2-methoxyestradiol protects against ischemia/reperfusion injury in alcoholic fatty liver by enhancing sirtuin 1-mediated autophagy. Biochem Pharmacol 131:40-51. doi:10.1016/j.bcp.2017.02.008

Cockburn BN, Coore HG (1995) Starvation reduces pyruvate dehydrogenase phosphate phosphatase activity in rat kidney. Mol Cell Biochem 149-150:131-136

Cully M, You H, Levine AJ, Mak TW (2006) Beyond PTEN mutations: the PI3K pathway as an integrator of multiple inputs during tumorigenesis. Nat Rev Cancer 6(3):184-192. doi:10.1038/ nrc1819

Erbil S, Oral O, Mitou G et al (2016) RACK1 is an interaction partner of ATG5 and a novel regulator of autophagy. J Biol Chem 291(32):16753-16765. doi:10.1074/jbc.M115.708081

Fiacco E, Castagnetti F, Bianconi V et al (2016) Autophagy regulates satellite cell ability to regenerate normal and dystrophic muscles. Cell Death Differ 23(11):1839-1849. doi:10.1038/ cdd. 2016.70

Garcia D, Shaw RJ (2017) AMPK: mechanisms of cellular energy sensing and restoration of metabolic balance. Mol Cell 66(6):789-800. doi:10.1016/j.molcel.2017.05.032

Gavand PE, Serio I, Arnaud L et al (2017) Clinical spectrum and therapeutic management of systemic lupus erythematosus-associated macrophage activation syndrome: a study of 103 episodes in 89 adult patients. Autoimmun Rev 16(7):743-749. doi:10.1016/j. autrev.2017.05.010

Hosokawa N, Sasaki T, Iemura S, Natsume T, Hara T, Mizushima N (2009) Atg101, a novel mammalian autophagy protein interacting with Atg13. Autophagy 5(7):973-979

Klionsky DJ, Codogno P, Cuervo AM et al (2010) A comprehensive glossary of autophagy-related molecules and processes. Autophagy 6(4):438-448. doi:10.4161/auto.6.4.12244

Lee CM, Tripathi S, Morgan ET (2017) Nitric oxide-regulated proteolysis of human CYP2B6 via the ubiquitin-proteasome system. Free Radic Biol Med 108:478-486. doi:10.1016/j. freeradbiomed.2017.04.015

Liang XH, Jackson S, Seaman M et al (1999) Induction of autophagy and inhibition of tumorigenesis by beclin 1. Nature 402(6762):672-676. doi:10.1038/45257

Lim JA, Li L, Shirihai OS, Trudeau KM, Puertollano R, Raben N (2017) Modulation of mTOR signaling as a strategy for the treatment of Pompe disease. EMBO Mol Med 9(3):353-370. doi:10.15252/emmm.201606547

Luo L, Dai JR, Guo SS et al (2017) Lysosomal proteolysis is associated with exercise-induced improvement of mitochondrial quality control in aged hippocampus. J Gerontol A Biol Sci Med Sci. doi: $10.1093 /$ gerona/glw242

Martin LJ, Gupta J, Jyothula SS et al (2012) Functional variant in the autophagy-related 5 gene promotor is associated with childhood asthma. PLoS One 7(4):e33454. doi:10.1371/journal. pone. 0033454 
Massey DC, Parkes M (2007) Genome-wide association scanning highlights two autophagy genes, ATG16L1 and IRGM, as being significantly associated with Crohn's disease. Autophagy 3(6):649-651

Mathiassen SG, De Zio D, Cecconi F (2017) Autophagy and the cell cycle: a complex landscape. Front Oncol 7:51. doi:10.3389/fonc.2017.00051

Mizushima N, Komatsu M (2011) Autophagy: renovation of cells and tissues. Cell 147(4):728-741. doi:10.1016/j.cell.2011.10.026

Morita S, Villalta SA, Feldman HC et al (2017) Targeting ABL-IRE1alpha signaling spares ERstressed pancreatic beta cells to reverse autoimmune diabetes. Cell Metab 25(4):883.e8-897.e8. doi:10.1016/j.cmet.2017.03.018

Nascimbeni AC, Fanin M, Angelini C, Sandri M (2017) Autophagy dysregulation in Danon disease. Cell Death Dis 8(1):e2565. doi:10.1038/cddis.2016.475

Nath S, Dancourt J, Shteyn V et al (2014) Lipidation of the LC3/GABARAP family of autophagy proteins relies on a membrane-curvature-sensing domain in Atg3. Nat Cell Biol 16(5):415-424. doi:10.1038/ncb2940

Niu Y, Sun W, Lu JJ et al (2016) PTEN activation by DNA damage induces protective autophagy in response to cucurbitacin B in hepatocellular carcinoma cells. Oxidative Med Cell Longev 2016:4313204. doi:10.1155/2016/4313204

Petherick KJ, Conway OJ, Mpamhanga C et al (2015) Pharmacological inhibition of ULK1 kinase blocks mammalian target of rapamycin (mTOR)-dependent autophagy. J Biol Chem 290(18):11376-83. doi:10.1074/jbc.C114.627778

Petibone DM, Majeed W, Casciano DA (2017) Autophagy function and its relationship to pathology, clinical applications, drug metabolism and toxicity. J Appl Toxicol 37(1):23-37. doi:10.1002/jat.3393

Pham DL, Kim SH, Losol P et al (2016) Association of autophagy related gene polymorphisms with neutrophilic airway inflammation in adult asthma. Korean J Intern Med 31(2):375-385. doi:10.3904/kjim.2014.390

Rivera JF, Costes S, Gurlo T, Glabe CG, Butler PC (2014) Autophagy defends pancreatic beta cells from human islet amyloid polypeptide-induced toxicity. J Clin Invest 124(8):3489-3500. doi:10.1172/JCI71981

Schwartz RA, Fernandez G, Kotulska K, Jozwiak S (2007) Tuberous sclerosis complex: advances in diagnosis, genetics, and management. J Am Acad Dermatol 57(2):189-202. doi:10.1016/j. jaad.2007.05.004

Sparks JD, O'Dell C, Chamberlain JM, Sparks CE (2013) Insulin-dependent apolipoprotein B degradation is mediated by autophagy and involves class I and class III phosphatidylinositide 3kinases. Biochem Biophys Res Commun 435(4):616-620. doi:10.1016/j.bbrc.2013.05.029

Stappenbeck TS, Rioux JD, Mizoguchi A et al (2011) Crohn disease: a current perspective on genetics, autophagy and immunity. Autophagy 7(4):355-374. doi:10.4161/auto.7.2.13074

Sumis A, Cook KL, Andrade FO et al (2016) Social isolation induces autophagy in the mouse mammary gland: link to increased mammary cancer risk. Endocr Relat Cancer 23(10):839-856. doi:10.1530/ERC-16-0359

Vescovo T, Refolo G, Vitagliano G, Fimia GM, Piacentini M (2016) Molecular mechanisms of hepatitis C virus-induced hepatocellular carcinoma. Clin Microbiol Infect 22(10):853-861. doi:10.1016/j.cmi.2016.07.019

Wang L, Khambu B, Zhang H, Yin XM (2015) Autophagy in alcoholic liver disease, self-eating triggered by drinking. Clin Res Hepatol Gastroenterol 39(Suppl 1):S2-S6. doi:10.1016/j. clinre.2015.05.023

Wang S, Pacher P, De Lisle RC, Huang H, Ding WX (2016) A mechanistic review of cell death in alcohol-induced liver injury. Alcohol Clin Exp Res 40(6):1215-1223. doi:10.1111/acer.13078

Wesselborg S, Stork B (2015) Autophagy signal transduction by ATG proteins: from hierarchies to networks. Cell Mol Life Sci 72(24):4721-4757. doi:10.1007/s00018-015-2034-8 
Xu Y, Zhou Q, Xin W, Li Z, Chen L, Wan Q (2016) Autophagy downregulation contributes to insulin resistance mediated injury in insulin receptor knockout podocytes in vitro. Peer $\mathrm{J} 4$ : e1888. doi:10.7717/peerj.1888

Yang L, Li P, Fu S, Calay ES, Hotamisligil GS (2010) Defective hepatic autophagy in obesity promotes ER stress and causes insulin resistance. Cell Metab 11(6):467-478. doi:10.1016/j. cmet.2010.04.005

Yilmaz E (2017) Endoplasmic reticulum stress and obesity. Adv Exp Med Biol 960:261-276. doi:10.1007/978-3-319-48382-5 11

Zhang Y, Goldman S, Baerga R, Zhao Y, Komatsu M, Jin S (2009) Adipose-specific deletion of autophagy-related gene 7 (atg7) in mice reveals a role in adipogenesis. Proc Natl Acad Sci U S A 106(47):19860-19865. doi:10.1073/pnas.0906048106

Zhong L, Shu W, Dai W, Gao B, Xiong S (2017) ROS-mediated JNK activation contributes to HBxinduced autophagy via regulating beclin-1/Bcl-2 interaction. J Virol. doi:10.1128/JVI.00001-17

Zhou XJ, Lu XL, Lv JC et al (2011) Genetic association of PRDM1-ATG5 intergenic region and autophagy with systemic lupus erythematosus in a Chinese population. Ann Rheum Dis 70(7):1330-1337. doi:10.1136/ard.2010.140111 